\title{
Kurze Ketten im Lebensmittelbereich
}

\section{Herausforderungen und Potenziale von verschiedenen Typen von Short Food Supply Chains}

\author{
Gesine Tuitjer ${ }^{1}$ \\ Eingegangen: 7. Dezember 2020 / Überarbeitet: 4. März 2021 / Angenommen: 5. März 2021 / Online publiziert: 1. April 2021 \\ (c) Der/die Autor(en) 2021
}

\section{Zusammenfassung}

Short Food Supply Chains (SFSC), also kurze Wertschöpfungsketten im Lebensmittelbereich, sind ein verbreiteter Ansatz in der Regionalentwicklung - im ländlichen wie auch im städtischen Kontext. Aufgrund der Breite des Konzeptes sind empirische Belege für die regionalen Auswirkungen von kurzen Ketten jedoch uneindeutig und ihrem konkreten Aufbau stehen teilweise große Herausforderungen gegenüber. Im vorliegenden Text werden Direktvermarktung, Regionalvermarktungsinitiativen und regionalisierte vertikale Wertschöpfungsketten behandelt. Der Text fasst Ergebnisse einzelner Fallstudien zusammen, die zwischen 2015 und 2019 in 6 ländlichen Kreisen durchgeführt wurden. Im Text werden Herausforderungen für die Entwicklung dieser kurzen Ketten skizziert und mögliche Chancen aufgezeigt. Der Ausbau von alternativen Vermarktungswegen kann beispielsweise durch Infrastruktur im Bereich der Lagerung und Logistik unterstützt werden. Ein weiteres zentrales Ergebnis stellt die Notwendigkeit von Netzwerkvermittlern dar, die Kooperationen zwischen Produzenten innerhalb einer Region entlang vertikaler Wertschöpfungsketten begleiten bzw. initiieren.

Schlüsselwörter Lokale Lebensmittelsysteme · Alternative Food Networks · Kurze Ketten · Regionale Wertschöpfung · Regionalvermarktungsinitiativen · Direktvermarktung

\begin{abstract}
In rural and urban areas alike, Short Food Supply Chains (SFSCs) are a common approach to regional development. Because the concept of SFSCs is rather wide, however, empirical evidence of the regional effects of SFSCs remains vague, and there are sometimes major challenges involved in setting up SFSCs. This text deals with direct selling, regional marketing initiatives and regional vertical value chains. The text summarizes the results of individual case studies that were conducted between 2015 and 2019 in six rural regions. The text outlines the challenges for the development of these SFSCs and demonstrates possible opportunities. For example, the development of alternative food supply routes can be supported through infrastructure in the areas of storage and logistics. Another important result is the need for network managers, who supervise and/or initiate cooperation between producers along the value chains within a region.
\end{abstract}

Keywords Local Foodsystems - Alternative Food Networks - Short chains · Regional development $\cdot$ Regional marketing initiatives $\cdot$ Direct selling

\section{Einleitung}

Kurze Ketten in der Lebensmittelversorgung erfahren zu-

Gesine Tuitjer

gesine.tuitjer@thuenen.de

1 Johann Heinrich von Thünen-Institut, Bundesforschungsinstitut für Ländliche Räume, Wald und Fischerei, Braunschweig, Deutschland nehmend Aufmerksamkeit in Projekten der Regionalentwicklung, stehen aber häufig auch vor Problemen in der Umsetzung.

Generell verbindet die vielen teils sehr unterschiedlichen Formen von Alternative Food Networks (AFN) (Marsden et al. 2000) das gemeinsame Ziel, den ,Wert“ von Le- 
bensmitteln neu zu definieren und diesen Wert „fair“ zu verteilen, um so eine Alternative zu der dominanten Marktlogik zu bieten (Whatmore et al. 2003). Alternative Food Networks ist dabei primär ein konzeptioneller Begriff, unter dem Alternativen zum ,industriellen“ oder „standardisierten“ Lebensmittelsystem gebündelt werden, das durch wachsende Skalenerträge und die Marktmacht der Supermärkte gekennzeichnet ist. Im Unterschied zu AFN beschreibt der Ausdruck Short Food Supply Chain (im Folgenden als kurze Kette übersetzt) hingegen eher die verschiedenen Organisationsformen dieser alternativen Erzeuger-Konsumenten-Beziehungen (Renting et al. 2003). Allerdings werden die Begriffe häufig synonym verwendet.

Kurzen Ketten wird die Fähigkeit zugesprochen, die räumlichen und sozialen Beziehungen zwischen Konsument*innen und Produzenten*innen, und damit indirekt auch zwischen Stadt und Land, neu zu ordnen (Renting et al. 2003) sowie die Wertschöpfung innerhalb einer Region zu erhöhen und durch verringerte Transportwege einen Beitrag zum Umweltschutz zu leisten.

\section{Was sind kurze Ketten?}

In der strengsten Definition geht es tatsächlich darum, eine direkte Beziehung zwischen Landwirten und Verbrauchern herzustellen, um so „Vertrauen“ (wieder-)herzustellen. Die Bedeutung von Vertrauen als Dimension von alternativen Lebensmittelsystemen verweist deutlich auf die Entstehung der Konzepte zu Zeiten der BSE- und anderer Lebensmittelskandale. Vertrauen oder „Nähe“ muss allerdings nicht ausschließlich über den direkten Kontakt zu den Produzent*innen, sondern kann auch über vertrauenswürdige Zwischenhändler oder Siegel/Zeichen etc. hergestellt werden. Marsden et al. (2000) sprechen hier davon, dass die Produkte die Verbraucher „embedded with information" (Marsden et al. 2000, S. 425) erreichen sollen, sodass die Konsument*innen Wissen beispielsweise über den Herstellungsprozess, aber auch über die Betriebsethik erlangen können. Wie Abb. 1 zeigt, können dementsprechend auch Biosiegel und Fair-Trade-Produkte eingeschlossen sein. Regionalvermarktungsinitiativen und Regionalsiegel nehmen hier eine mittlere Position ein, da sowohl Formen der Direktvermarktung als auch Zertifizierungssysteme zur Anwendung kommen.

Bereits 2011 hat Tregear darauf hingewiesen, dass das sehr weite und normativ fundierte Konzept der ,alternativen" Lebensmittelnetzwerke die empirische Erforschung der tatsächlichen regional-ökonomischen und ökologischen Effekte erschwert. Denn die Opportunitäts- und Transaktionskosten für Erzeuger*innen wie auch für Konsument*innen sind mitunter hoch (Kögl und Tietze 2010; Tregear 2011; Vogt et al. 2016), nicht jede Alternative zum konventionellen Lebensmittelsystem ist nachhaltig bzw. reduziert den $\mathrm{CO}_{2}$-Ausstoß (Coley et al. 2009) und Direktvermarktung und Regionalsiegel können Verdrängungseffekte hervorrufen (Ilbery und Maye 2006; Hughes und Boys 2015; Neill et al. 2020), was zum Auseinanderfallen von einzelbetrieblichem und regionalem Nutzen führt.

In der Praxis der Regionalentwicklung spielen kurze Ketten und regionale Lebensmittel nichtsdestotrotz eine Rolle, die mit Blick auf die Farm-to-Fork-Strategie (EU 2020) zukünftig vermutlich noch größer werden wird.

Vor diesem Hintergrund möchte der folgende Text die typischen Schwierigkeiten und Potenziale, die mit verschiedenen Formen von kurzen Ketten verknüpft sind, gebündelt darstellen. Dazu wird auf den Stand der Forschung zu einzelnen Formen von kurzen Ketten und die Ergebnisse verschiedener empirischer Fallstudien zurückgegriffen. Der folgende Abschnitt stellt den Projekthintergrund und die Datengrundlage dar, daran anschließend werden 3 Beispiele für kurze Ketten präsentiert.
Abb. 1 Beispiele für ,kurze Ketten“

\section{Beispiele für ,kurze Ketten“}

\begin{tabular}{|c|c|c|c|}
\hline $\begin{array}{l}\text { Soziale Nähe \& } \\
\text { Vertrauen }\end{array}$ & $\begin{array}{l}\text { Direktvermarktung } \\
\text { beispielsweise } \\
\text { - Solawi/CSA } \\
\text { - Bauernmärkte } \\
\text { - Hofläden } \\
\text { - Abo-Kisten etc. }\end{array}$ & $\begin{array}{l}\text { Regionalvermarktungs- } \\
\text { initiativen }\end{array}$ & $\begin{array}{l}\text {,Alternative'Siegel } \\
\text { \& Zertifikate } \\
\text { beispielsweise } \\
\text { - } \quad \text { Fairtrade } \\
\text { - Bio } \\
\text { - EU-Qualitätssiegel: } \\
\text { geschützte geografische } \\
\text { Angabe, geschützte Ur- } \\
\text { sprungsbezeichnung \& } \\
\text { garantiert traditionelle } \\
\text { Spezialität }\end{array}$ \\
\hline
\end{tabular}


Tab. 1 Daten und Methode der Teilstudien. (Quelle: eigene Darstellung)

\begin{tabular}{lll}
\hline Region(en) & Ziel der Projekte & Daten und Methode \\
\hline Greiz & $\begin{array}{l}\text { Aufbau eines Regionalwarenlagers und Ausbau } \\
\text { eines Abo-Systems }\end{array}$ & $\begin{array}{l}\text { Fallstudie, } 4 \text { Expert*inneninterviews, 3 } \\
\text { teilnehmende Beobachtungen }\end{array}$ \\
Sigmaringen, Elbe-Elster, Höxter, Mittel- & $\begin{array}{l}\text { Auf-/Ausbau einer } \\
\text { sachsen, Neunkirchen }\end{array}$ & $\begin{array}{l}\text { Regionalvermarktungsinitiative } \\
\text { Vorport*inneninterviews mit den Lei- } \\
\text { ter*innen der Initiativen }\end{array}$ \\
& $\begin{array}{l}\text { Aufbau regionaler Wertschöpfungsketten und } \\
\text { Lebensmittelmanufakturen }\end{array}$ & Fallstudie, 11 Expert*inneninterviews
\end{tabular}

\section{Projekthintergrund und Datengrundlage}

Der vorliegende Text bündelt die Ergebnisse einzelner Fallstudien zum Aufbau von kurzen Ketten. Die zugrundeliegenden Projekte wurden im Rahmen des Bundesmodellvorhabens Land(auf)Schwung zwischen 2015 und 2019 gefördert. Das Modellvorhaben hatte u. a. zum Ziel, innovative Wege zur Steigerung der regionalen Wertschöpfung zu erproben.

Das ausgewertete Material umfasst eine Fallstudie zum Aufbau einer Öko-Kiste in einem Landkreis in Thüringen. Ferner wurden die Leiter*innen von 6 Regionalvermarktungsinitiativen (10 Interviews) interviewt, die sich zwischen 2015 und 2019 im Aufbau oder in der Weiterentwicklung befanden. Weiterhin wurde eine Fallstudie zum Aufbau regionalisierter Wertschöpfungsketten in MecklenburgVorpommern durchgeführt, für die 11 Personen interviewt wurden. Im Rahmen dieses Projektes sollten 3 verschiedene vertikale Wertschöpfungsketten und Kooperationen über verschiedene Betriebsgrößen hinweg etabliert werden. Die Fallstudien wurden im Rahmen der Begleitforschung für dieses Bundesmodellvorhaben durch die Autorin durchgeführt (Tab. 1).

Die Interviews wurden leitfadengestützt erhoben und inhaltsanalytisch ausgewertet (Mayring 2003). Die Interviews thematisierten den Projektverlauf und insbesondere die Schwierigkeiten, Herausforderungen und besonderen Erfolge der Projekte.

\section{Ergebnisse}

\section{Herausforderungen im Aufbau von kurzen Ketten am Beispiel der Direktvermarktung}

Direktvermarktung spielt insbesondere im Ökolandbau eine wachsende Rolle (Böhm und Krämer 2020, S. 32). Digitale und kooperative Formen bieten zunehmend Chancen für die Direktvermarktung. Dies sind beispielsweise Abo-Modelle oder die solidarische Landwirtschaft (Wellner und Theuvsen 2017) sowie durch Dritte organisierte Vermarktungsangebote wie Marktschwärmer (Recke et al. 2020). Über diese Wege kann die Wertschöpfung insbesondere für kleine und spezialisierte landwirtschaftliche Be- triebe gesteigert werden. Als größte Herausforderung für die Direktvermarktung weisen verschiedene standardisierte Befragungen unter Erzeuger*innen insbesondere die hohe Arbeitsbelastung und Kosten für Transport und Verpackung aus (Kögl und Tietze 2010; Böhm und Krämer 2020).

Im Rahmen der Fallstudie wurde ein Hof untersucht, der ursprünglich in der einzelbetrieblichen Direktvermarktung (Abo-Kisten) aktiv war. Hieraus entstand letztendlich ein regionaler Erzeugerzusammenschluss, der Abo-Kisten an die Konsument*innen der Region ausliefert. Durch das Fördervorhaben erhielten die Gründer*innen Zuschüsse für den Hofumbau zu einem Logistikzentrum mit Kühl- und Lagerräumen und Warenwirtschaftssystem. Im Zuge der Projektlaufzeit konnten sowohl der Kreis der regionalen Produzent*innen, die ihre Waren unter dem Dach des $\mathrm{Zu}$ sammenschlusses vermarkten, wie auch der Radius, in dem beliefert wird, deutlich ausgeweitet werden. Der Zusammenschluss bietet mittlerweile auch Kochboxen an (Lieferung der portionierten Waren inklusive Zubereitungsanleitung) und beliefert den Landkreis mit Schulobst (Abb. 2).

In der Fallstudie hat die vorhandene Infrastruktur insbesondere den Ausbau eines Netzwerks befördert, da den neu hinzukommenden Erzeuger*innen konkrete Angebote zum Vertrieb ihrer Waren gemacht werden konnten. Darüber hinaus wurden neu entstandene Lebensmittelmanufakturen im Rahmen der Förderung ermuntert, Teil der Vermarktungsgemeinschaft zu werden. Außerdem konnte durch die Lieferung von Schulobst und portionierten Waren in regionaler Bioqualität eine Nische bedient und ein Alleinstellungsmerkmal geschaffen werden.

\section{Herausforderungen im Aufbau von kurzen Ketten am Beispiel von Regionalmarken, Regionalsiegel und regionalen Vermarktungsinitiativen}

Die Auszeichnung regionaler Produkte per Siegel oder Regionalmarke und die Entwicklung einer Regionalvermarktungsinitiative kann ein Weg sein, Wertschöpfung in der Region zu fördern. Allerdings sind gerade bei den kleinen Mengen sehr heterogener Waren, die produziert werden, Lagerung und Logistik verhältnismäßig teuer (Vogt et al. 2016, S. 5) und der Gebietszuschnitt darf nicht zu klein gewählt werden, um mittelfristig Wachstum zu ermöglichen. Ebenso stehen Kreise bzw. Regionen vor Her- 


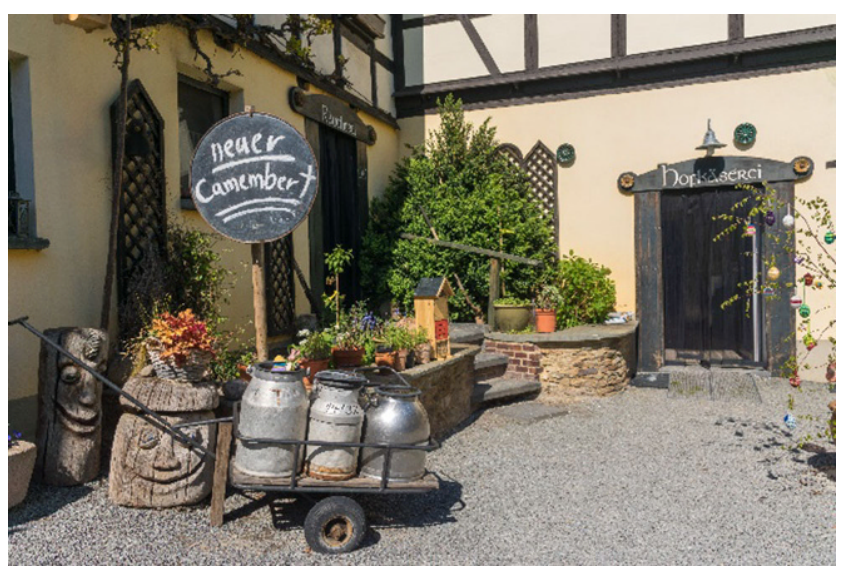

Abb. 2 Vierseithof mit Käserei und Lager. (Copyright: Hofkäserei)

ausforderungen, die nicht durch landwirtschaftliche bzw. kleinteilige landwirtschaftliche Strukturen und eine gewisse Angebotsbreite geprägt sind. Insbesondere die Transaktionskosten, die mit dem Aufbau eines Netzwerkes sowie mit der Etablierung einer regionalen Marke verbunden sind, sind hoch. Gleichzeitig bietet der Zusammenschluss von Erzeuger*innen gegenüber der einzelbetrieblichen Direktvermarktung auch Chancen, wenn durch intensive Kooperationen der Austausch von Wissen und Ressourcen befördert und mittelfristig Innovationen und Wachstum in der Region unterstützt werden.

In der Fallstudie wurde die Entwicklung von 6 Regionalvermarktungsinitiativen untersucht. Im Rahmen der Interviews erwiesen sich insbesondere die Anzahl an potenziellen Mitgliedern und deren Bereitschaft zur Teilnahme sowie die (Kostenverteilung der) Logistik als Knock-outKriterien. Im Landkreis Elbe-Elster entstand eine Regionalvermarktungsinitiative, die unter dem Logo bzw. dem Siegel des Landkreises Produkte auszeichnet. Das Projekt wurde 2016 initiiert und seitdem sind knapp 40 Betriebe im Landkreis Zeichenträger geworden. Hier sind zu-

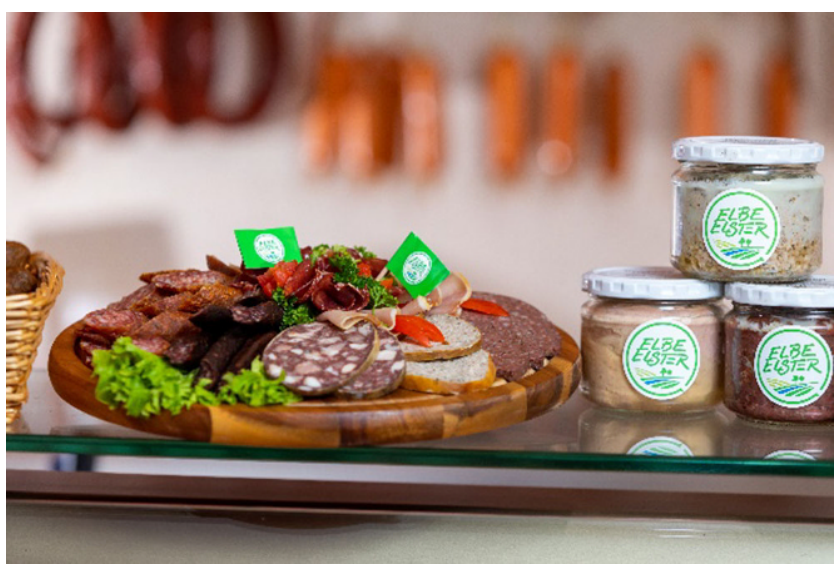

Abb. 3 Regionalsiegel Elbe-Elster. (Copyright: Fleischerei Weiland) sätzlich zum Zertifizierungssystem ein privatwirtschaftlicher Lieferdienst und ein stationärer Laden im Stadtzentrum sowie Abnahmebeziehungen mit kreiseigenen Großabnehmern (Krankenhaus, Kindergarten) entstanden. Das Gros der Produkte wird jedoch über die individuellen Produktionsstätten vertrieben. Initiiert wurde dieses Projekt durch die Kreiswirtschaftsförderung, die ebenfalls Netzwerktreffen organisiert hat. Im Rahmen dieser Treffen konnten Kooperationen zwischen den Kleinstbetrieben aufgebaut werden, aus denen heraus neue Produkte entwickelt wurden. Durch Kooperationen lässt sich zum Teil auch die Auslastung der einzelnen Verarbeitungsmaschinen verbessern (Abb. 3).

\section{Herausforderungen im Aufbau von kurzen Ketten am Beispiel von vertikalen Wertschöpfungsketten}

Die Regionalisierung vorhandener Wertschöpfungsketten im „konventionellen“ Lebensmittelsystem hat sicherlich großes transformatives Potenzial. Beim Aufbau von Kooperationen über verschiedene Wertschöpfungsstufen und damit ggf. verbundenen Produktionsgrößen und -logiken hinweg entstehen jedoch verhältnismäßig hohe Transaktionskosten. Häufig passen regionales Angebot und regionale Nachfrage nicht zusammen (Schultz und Scherer 1997; Vogt et al. 2016). Weitere übliche Hindernisse sind fehlende Zwischenverarbeiter, beispielsweise Molkereien oder Mühlen, die die regional erzeugten Rohstoffe für die Weiterverarbeitung aufbereiten (Vogt et al. 2016). Schlussendlich ist die regionale Beschaffung von Rohstoffen für Verarbeiter häufig aufwendig und wird dementsprechend eher im Spezialitäten- oder Nischensegment als Alleinstellungsmerkmal genutzt (Maier et al. 2020). Die Evaluation verschiedener Länderprogramme zeigt dementsprechend, dass Förderung für den Aufbau regionalisierter Wertschöpfungsketten nur in sehr geringem Maße nachgefragt wird (Forstner 2020; Spengler und Schramek 2020).

Im Rahmen der Fallstudie wurden Interviews mit insgesamt 5 kleinen Lebensmittelmanufakturen in Vorpommern-Rügen geführt, die im Aufbauprozess der Betriebe sowohl Möglichkeiten für die regionale Beschaffung von Rohstoffen als auch für die regionale Weiterverarbeitung in Kooperation mit Großbetrieben der Lebensmittelindustrie ausgelotet haben. Unabhängig von ggf. divergierendem Angebot und Nachfrage nach regionalen Produkten hat die Fallstudie ergeben, dass Kooperationen bzw. vertikale Beziehungen zwischen Betrieben verschiedener Größe und/ oder Branchen teilweise durch inkompatible betriebliche Logiken beeinträchtigt werden. Diese inkompatiblen Produktionslogiken betreffen beispielsweise Wert- und Normvorstellungen über den Produktionsprozess eines Produktes oder die Produkteigenschaften. In ähnlicher Form müssen nicht nur Differenzen aufgrund von Größe und Verarbei- 
tungsmenge, sondern auch ideologische Differenzen zwischen „,konventionellen“ und ,alternativen“ Lebensmittelproduzent*innen überwunden werden.

\section{Zusammenfassung der Ergebnisse und Fazit}

Für den Erfolg der geförderten, neuentstandenen Projekte hat sich das Vorhandensein bzw. die Förderung von Infrastrukturen (Lagerräume, Kühlzellen etc.) sowie die Begleitung des Netzwerkaufbaus durch zentrale Akteure der Region erwiesen. Die Regionalisierung etablierter Wertschöpfungsketten hingegen ist herausfordernder bzw. muss größere Opportunitätskosten überwinden als der Aufbau von alternativen Parallelstrukturen der Lebensmittelversorgung. Dementsprechend muss der Aufbau vertikaler regionaler Wertschöpfungsketten vor allem intensiv begleitet werden. Es bedarf auf der regionalen Ebene daher Akteure, die in der Lage sind, gegenseitiges Verständnis herzustellen und Vorteile für beide Seiten in der Kooperation deutlich zu machen - beispielsweise in Kommunikations- und Marketingeffekten durch die Verarbeitung regionaler Rohstoffe. Ein weiterer Ansatz, wie regionale Wertschöpfungsketten und Unternehmertum unterstützt werden können, liegt in der Förderung von Zwischenverarbeitern wie Molkereien, Mühlen und Mälzereien (Vogt et al. 2016).

Der vorliegende Text sollte zeigen, dass ,kurze Ketten“ als regionale Parallelstruktur teils mit hohen Transaktionsund Opportunitätskosten für Produzent*innen (und Konsument*innen) verbunden sind. Daher werden sie hier auch nicht als Lösung der Probleme des Agrar- und Ernährungssystems, sondern vielmehr als Wegbereiter für die Transformation $\mathrm{zu}$ einem insgesamt nachhaltigen ,,konventionellen" System gesehen. Erste Schritte in diese Richtung, nach jahrzehntelangen Anreizen für Wachstum und Konzentration, können - mit dem nötigen Optimismus - in der Farmto-Fork-Strategie der EU gesehen werden, die als Bestandteil des „Green Deals“ Aspekte wie nachhaltige Landwirtschaft, Ernährungssicherheit und Klimaschutz zusammenbringt (EU 2020, S. 15). Allerdings zeigt sich auch hier das Spannungsverhältnis zwischen dem Aufbau eines nachhaltigen Parallelsystems und der Transformation des konventionellen Systems. So mahnt der Sachverständigenrat Ländliche Entwicklung: „Insbesondere sollten diese Strategien eine stärkere Berücksichtigung in der ersten Säule der Gemeinsamen Agrarpolitik finden mit dem Ziel, das erhebliche Fördervolumen im Bereich der Direktzahlungen für eine entsprechend umweltgerechtere Ausrichtung der Landwirtschaft zu nutzen“ (SRLE 2021, S. 9).

Funding Dieses Projekt wurde durch das Bundesministerium für Ernährung und Landwirtschaft, Förderkennzeichen BULE 2815LE007, gefördert.
Funding Open Access funding enabled and organized by Projekt DEAL.

Open Access Dieser Artikel wird unter der Creative Commons Namensnennung 4.0 International Lizenz veröffentlicht, welche die Nutzung, Vervielfältigung, Bearbeitung, Verbreitung und Wiedergabe in jeglichem Medium und Format erlaubt, sofern Sie den/die ursprünglichen Autor(en) und die Quelle ordnungsgemäß nennen, einen Link zur Creative Commons Lizenz beifügen und angeben, ob Änderungen vorgenommen wurden.

Die in diesem Artikel enthaltenen Bilder und sonstiges Drittmaterial unterliegen ebenfalls der genannten Creative Commons Lizenz, sofern sich aus der Abbildungslegende nichts anderes ergibt. Sofern das betreffende Material nicht unter der genannten Creative Commons Lizenz steht und die betreffende Handlung nicht nach gesetzlichen Vorschriften erlaubt ist, ist für die oben aufgeführten Weiterverwendungen des Materials die Einwilligung des jeweiligen Rechteinhabers einzuholen.

Weitere Details zur Lizenz entnehmen Sie bitte der Lizenzinformation auf http://creativecommons.org/licenses/by/4.0/deed.de.

\section{Literatur}

Böhm M, Krämer C (2020) Schlussbericht zum Thema „Neue und innovative Formen der Direktvermarktung landwirtschaftlicher Produkte - Analyse und Erarbeitung von Handlungsempfehlungen“ (Freising: ECOZEPT GbR). https://www.ble.de/SharedDocs/ Pressemitteilungen/DE/2020/200311_Direktvermarktung.htm. Zugegriffen: 4. Febr. 2021

Coley D, Howard M, Winter M (2009) Local food, food miles and carbon emissions: a comparison of farm shop and mass distribution approaches. Food Policy 34:150-155. https://doi.org/10.1016/ j.foodpol.2008.11.001

EU (2020) Mitteilung der Kommission an das Europäische Parlament, den Europäischen Rat, den Rat, den Europäischen Wirtschaftsund Sozialausschuss und den Ausschuss der Regionen „Vom Hof auf den Tisch“ - eine Strategie für ein faires, gesundes und umweltfreundliches Lebensmittelsystem, COM/2020/381 final

Forstner B (2020) Evaluierung der Förderung von Investitionen im Bereich der Verarbeitung und Vermarktung landwirtschaftlicher Erzeugnisse (Schwerpunktbereich 3A): Landesprogramm ländlicher Raum (LPLR) des Landes Schleswig-Holstein 2014 bis 2020. DE: Johann Heinrich von Thünen-Institut. https://doi.org/ 10.3220/5LE1588237728000

Hughes D, Boys K (2015) What we know and don't know about the economic development benefits of local food systems. Choices Mag Food Farm Resour Issues 301:1-6

Ilbery B, Maye D (2006) Retailing local food in the scottish-english borders: a supply chain perspective. Geoforum 37:352-367

Kögl H, Tietze J (2010) Regionale Erzeugung, Verarbeitung und Vermarktung von Lebensmitteln (Universität Rostock)

Maier P, Klein O, Schumacher KP (2020) Alternative Zuliefersysteme im Brauereisektor - Regionalmodelle des Braugerstenbezugs in Bayern. Standort. https://doi.org/10.1007/s00548-020-00682-7

Marsden T, Banks J, Bristow G (2000) Food supply chain approaches: exploring their role in rural development. Sociol Ruralis 40:424-438

Mayring P (2003) Qualitative Inhaltsanalyse. Grundlagen und Techniken, 6. Aufl. Beltz,

Neill CL, Holcomb RB, Lusk JL (2020) Estimating potential beggarthy-neighbor effects of state labeling programs. Agribusiness 36:3-19

Recke G, Janßen M, Albers A (2020) Innovative digitale Formen der Direktvermarktung am Beispiel Marktschwärmer - Potenziale 
und Risiken. In: Digitalisierung für Mensch, Umwelt und Tier Referate der 40. GIL-Jahrestagung Weihenstephan, S 253-258

Renting H, Marsden T, Banks J (2003) Understanding alternative food networks: exploring the role of short food supply chains in rural development. Environ Plan A 35(3):393-411. https://doi.org/10. 1068/a3510

Schultz B, Scherer R (1997) Regionalvermarktung in Großschutzgebieten Leitlinien und Fallbeispiele. EURES-Institut, Freiburg

Spengler B, Schramek J (2020) Ad hoc-Studie zur Verbesserung der Akzeptanz der Vorhabensart M 6.4b - Förderung von Investitionen in die Verarbeitung und Vermarktung regionaler Erzeugnisse in regionalen Wertschöpfungsketten. Im Auftrag des Ministerium für Wirtschaft, Verkehr, Landwirtschaft und Weinbau (MWVLW) Referat 8605 - Investitionsförderung, Förderung der Vermarktung und ländlicher Entwicklungsmaßnahmen. IfLS, Frankfurt am Main. https://www.ifls.de/referenzen/publikationen/details/adhoc-studie-zur-verbesserung-der-akzeptanz-der-vorhabensartm-64b-foerderung-von-investitionen-i/. Gesehen 4. Febr. 2021

SRLE - Sachverständigenrat Ländliche Entwicklung beim Bundesministerium für Ernährung und Landwirtschaft (2021) Kraftvolle ländliche Räume für ein starkes Europa: Beitrag zur Debatte über eine „Langfristige Vision für die ländlichen Räume“ der
Europäischen Kommission. https://www.bmel.de/SharedDocs/ Downloads/DE/_Ministerium/Beiraete/srle/stellungnahme-srle-

vision-1\%C3\%A4ndliche-r\%C3\%A4ume.pdf?_blob=publication File \&v=2. Zugegriffen: 4. Febr. 2021

Tregear A (2011) Progressing knowledge in alternative and local food networks: critical reflections and a research agenda. J Rural Stud 27:419-430

Vogt L, Schütz K, Mergenthaler M (2016) Regionalisierung von Lieferketten in der Ernährungswirtschaft - Herausforderungen und Ansatzpunkte. Eine Falluntersuchung am Beispiel des Kreises Höxter (Nordrhein-Westfalen). 39. Forschungsberichte des Fachbereichs Agrarwirtschaft, Soest. https://doi.org/10.13140/RG.2.1. 2050.3449

Wellner M, Theuvsen L (2017) Landwirtschaft von unten: Community Supported Agriculture als zivilgesellschaftliche Nachhaltigkeitsinitiative. In: Theuvsen L, Andeßner R, Gmür M, Greiling D (Hrsg) Nonprofit-Organisationen und Nachhaltigkeit. Springer, Wiesbaden, S 235-244

Whatmore S, Stassart P, Renting H (2003) What's alternative about alternative food networks. Environ Plan A 35:389-3391. https:// doi.org/10.1068/a3621 